Preprint de "A maximal domain of preferences for strategy-proof, efficient, and simple rules in the division problem," de Jordi Massó i Alejandro Neme. Social Choice and Welfare 23, 187-206 (2004). Lliurat a Springer l'abril de 2004. 


\title{
A Maximal Domain of Preferences for Strategy-proof, Efficient, and Simple Rules in the Division Problem*
}

\author{
Jordi Massó ${ }^{\dagger}$ and Alejandro Neme ${ }^{\ddagger}$
}

April 2003

\begin{abstract}
The division problem consists of allocating an amount $M$ of a perfectly divisible good among a group of $n$ agents. Sprumont (1991) showed that if agents have single-peaked preferences over their shares, the uniform rule is the unique strategy-proof, efficient, and anonymous rule. Ching and Serizawa (1998) extended this result by showing that the set of single-plateaued preferences is the largest domain, for all possible values of $M$, admitting a rule (the extended uniform rule) satisfying strategy-proofness, efficiency and symmetry. We identify, for each $M$ and $n$, a maximal domain of preferences under which the extended uniform rule also satisfies the properties of strategyproofness, efficiency, "tops-onlyness", and continuity. These domains (called partially single-plateaued) are strictly larger than the set of single-plateaued preferences. However, their intersection, when $M$ varies from zero to infinity, coincides with the set of single-plateaued preferences.
\end{abstract}

Journal of Economic Literature Classification Numbers: D71, D78, D63.

Keywords: Strategy-proofness, single-plateaued preferences.

*An earlier version of this paper circulated under the title "A maximal domain of preferences for tops-only rules in the division problem". We are grateful to an associate editor of this journal for comments that helped to improve the presentation of the paper and to Matt Jackson for suggesting us the interest of identifying a maximal domain of preferences for tops-only rules. We are also grateful to Dolors Berga, Flip Klijn, Howard Petith, and a referee for helpful comments. The work of Alejandro Neme is partially supported by Research Grant 319502 from the Universidad Nacional de San Luis. The work of Jordi Massó is partially supported by Research Grants BEC2002-02130 from the Spanish Ministerio de Ciencia y Tecnología, and 2001SGR-00162 from the Generalitat de Catalunya. The paper was partially written while Alejandro Neme was visiting the UAB under a sabbatical fellowship from the Generalitat de Catalunya.

†Departament d'Economia i d'Història Econòmica and CODE. Universitat Autònoma de Barcelona. 08193, Bellaterra (Barcelona), Spain. E-mail: jordi.masso@uab.es.

¥Instituto de Matemática Aplicada de San Luis. Universidad Nacional de San Luis and CONICET. Ejército de los Andes 950. 5700, San Luis, Argentina. E-mail: aneme@unsl.edu.ar. 


\section{Introduction}

The division problem consists of allocating an amount $M$ of a perfectly divisible good among a group of $n$ agents. A rule maps preference profiles into $n$ shares of the amount $M$. Sprumont (1991) shows that, given $M$, if agents have single-peaked preferences over their shares, the uniform rule is the unique strategy-proof, efficient, and anonymous rule. This is a nice example of a large literature that, by restricting the domain of preferences, investigates the possibility of designing strategy-proof rules. ${ }^{1}$ Moreover, in this case, single-peakedness does not only allow strategy-proof rules but also efficient ones.

Whether or not nontrivial strategy-proof rules exist depends on the domain of preferences where we want them to operate. However, by restricting sufficiently the set of preferences it would always be possible to design non-dictatorial strategyproof rules for any environment. Therefore, and once a domain restriction has been identified (as one under which there are non-dictatorial strategy-proof rules), it is natural and meaningful to ask how much this domain can be enlarged to still allow for non-dictatorial strategy-proof rules. The Gibbard-Satterthwaite Theorem says that this maximal domain is strictly smaller than the universal domain of preferences.

Ching and Serizawa (1998) show that, when the rule depends not only on preferences but also on the amount $M$ to be allocated, the maximal domain under which there exists at least one rule (the extended uniform rule) satisfying strategyproofness, efficiency, and symmetry coincides with the set of single-plateaued preferences.

In Massó and Neme (2001) we show that, given $M$, the set of feebly singleplateaued preferences is the unique maximal domain of preferences that includes the set of single-peaked ones for which there exists at least one rule satisfying strategyproofness, efficiency, and strong symmetry. This set is strictly larger than the set of single-plateaued preferences; in particular, preferences might have special intervals of indifference away from the set of best shares. However, the rule that we exhibit when showing our maximality result is very complex and hence difficult to be implemented because it is not "tops-only" (it does not exclusively depend on the $n$ sets of best shares). Efficiency and strong symmetry force the rule to be sensitive to intervals of indifference away from the "top".

In this paper we ask how much the set of single-peaked preferences can be en-

\footnotetext{
${ }^{1}$ See Sprumont (1995), Barberà (1996), and Barberà (2001) for three comprehensive surveys of this literature as well as for three exhaustive bibliographies.
} 
larged to still allow for strategy-proof, efficient, and simple rules. In particular, we identify a maximal domain of preferences (that includes the set of single-peaked ones) for which there exists at least one rule (the extended uniform rule) satisfying strategy-proofness, efficiency, tops-onlyness, and continuity. We refer to this domain as the set of partially single-plateaued preferences. It turns out that this maximal domain depends crucially on both $M$ and $n$, and contains (for each value of $M$ ) the set of single-plateaued preferences. Moreover, the intersection of all sets of partially single-plateaued preferences, when $M$ varies from zero to infinity, coincides with the set of single-plateaued preferences. Notice that in Ching and Serizawa (1998) $M$ is treated as a variable of the problem rather than one of its data. We want to emphasize though that, in spite of their result, our analysis with a fixed amount $M$ is meaningful since there are many allocation problems where to assume the contrary would be senseless. Furthermore, we do not claim that the domain identified here has economic relevance per se; rather, we understand our result as giving a precise and definite answer to an interesting and economically relevant question.

The result here differs from our previous one in Massó and Neme (2001) on at least three grounds. The first difference has to do with the list of properties a rule is required to satisfy on the maximal domain. Instead of requiring strong symmetry we demand now that the rule be simple (i.e., tops-only and continuous). Any property related with anonymity is inappropriate when there are asymmetries among the agents that one wishes to respect (due to repeated relationships, seniority, etc.). ${ }^{2}$ On the other hand, tops-onlyness and continuity are two natural properties to ask for if we want to exclude complex rules that would be difficult to use. Second, while in Massó and Neme (2001) we identify the unique maximal domain containing the set of single-peaked preferences here we only identify a maximal domain. The reason why this difference arises is that on the domain of single-peaked preferences there is only one rule (the uniform one) satisfying strategy-proofness, efficiency, and strong symmetry while there are many that satisfy strategy-proofness, efficiency, tops-onlyness, and continuity; the extension may now be dependent upon the rule, yielding different maximal domains for different rules (in the last section of the paper we elaborate on this point by means of an example). Third, the two maximal domains are different; in particular, the set of feebly single-plateaued preferences strictly contains the set of partially single-plateaued preferences.

A number of papers have also identified maximal domains of preferences allowing

\footnotetext{
${ }^{2}$ See Barberà, Jackson, and Neme (1997) for many justifications of the use of non-anonymous rules.
} 
for strategy-proof social choice functions in voting environments. Barberà, Sonnenschein, and Zhou (1991) show that the set of separable preferences is the maximal domain that preserves strategy-proofness of voting by committees without dummies and vetoers. Serizawa (1995), Berga (1997), Barberà, Massó, and Neme (1999), Berga and Serizawa (2000), and Berga (2002) improve upon this result in several directions; for instance, either by looking at a more general voting model and/or by admitting larger classes of social choice functions.

In all papers mentioned above either the assumption of anonymity (or any other related property like symmetry, strong symmetry, or equal treatment of equals) and/or the assumption that the domain contains the set of single-peaked preferences guarantee that, on a subdomain of preferences, the outcome of the rule is unambiguously determined (by the uniform rule in the division problem and by a generalized median voter scheme in voting environments). ${ }^{3}$ And this supports very much the process of identifying the maximal domain of preferences. In contrast, we do not require here that the rule satisfy any property related to anonymity; ${ }^{4}$ therefore, even for single-peaked preference profiles, we do not know their associated vectors of shares since there are many rules satisfying the required properties yielding different outcomes. This is precisely one of our main difficulties here, which we overcome by, first, enlarging the set of single-plateaued preferences. We identify an intermediate domain (the set of weakly single-plateaued preferences) under which we can establish an anonymity like property of any rule defined on a larger domain satisfying the properties of strategy-proofness, efficiency, tops-onlyness, and continuity (Proposition 2). At the end of the paper we will comment on this apparent anonymity property implied by Proposition 2. Second, we show that preferences in the maximal domain (containing the set of weakly single-plateaued preferences) must have a weak monotonicity property (parts (a) and (b) of Proposition 3) on relevant intervals (between the share $\frac{M}{n}$ and the "top"). Finally, we obtain the maximal domain by identifying exactly where this monotonicity property must be strict (Lemmata 1 and 2 in the proof of Theorem 1).

The paper is organized as follows. Section 2, which closely follows Massó and Neme (2001), contains preliminary notation and definitions. The set of partially

\footnotetext{
${ }^{3}$ Theorem 1 in Berga and Serizawa (2000) assumes only that the domain of preferences contains a "minimally rich domain" in the sense that for each alternative $a$, there is at least one preference whose top is $a$.

${ }^{4}$ See Barberà, Jackson, and Neme (1997) to understand the difficulties of characterizing, in the division problem, non-anonymous rules on the domain of single-peaked preferences.
} 
single-plateaued preferences and the results are presented in Section 3. Section 4 concludes with some final remarks. An Appendix contains all proofs.

\section{Preliminary Notation and Definitions}

Agents are indexed by the elements of a finite set $N=\{1, \ldots, n\}$ where $n \geq 2$. They have to share the amount $M \in \mathbb{R}_{++}$of a perfectly divisible good. An allocation is a vector $\mathbf{x}=\left(x_{1}, \ldots, x_{n}\right) \in \mathbb{R}_{+}^{n}$ such that $\sum x_{i}=M$. We denote by $Z$ the set of allocations. Each agent has a complete preorder $R$ over $[0, M]$, his preference relation. Let $P$ be the strict preference relation associated with $R$ and let $I$ be the corresponding indifference relation. We assume that preferences are continuous in the sense that for each $x \in[0, M]$ the sets $\{y \in[0, M] \mid x R y\}$ and $\{y \in[0, M] \mid y R x\}$ are closed. We denote by $\mathcal{R}$ the set of continuous preferences on $[0, M]$ and by $\mathcal{V}$ a generic subset of $\mathcal{R}$. Given a preference relation $R \in \mathcal{R}$ we denote the set of preferred shares according to $R$ as $t(R)=\{x \in[0, M] \mid x R y$ for all $y \in[0, M]\}$. Let $\underline{t}(R)=$ $\min \{x \in[0, M] \mid x \in t(R)\}$ and $\bar{t}(R)=\max \{x \in[0, M] \mid x \in t(R)\}$. Abusing notation, we also denote by $t(R)$ the unique element of the set $t(R)$ whenever $\underline{t}(R)=\bar{t}(R)$. We call it the top of the preference relation $R$.

Preference profiles are $n$-tuples of continuous preference relations over $[0, M]$ and they are denoted by $\mathbf{R}=\left(R_{1}, \ldots, R_{n}\right) \in \mathcal{R}^{n}$. When we want to stress the role of agent $i$ 's preference we will represent a preference profile $\mathbf{R}$ by $\left(R_{i}, \mathbf{R}_{-i}\right)$. Given a preference profile $\mathbf{R}$ and a preference relation $R \neq R_{i}$ we will write $\left(R, \mathbf{R}_{-i}\right)$ to represent the preference profile where $R_{i}$ in $\mathbf{R}$ is replaced by $R$.

A rule on $\mathcal{V}^{n} \subseteq \mathcal{R}^{n}$ is a function $\Phi: \mathcal{V}^{n} \longrightarrow Z$; that is, $\sum \Phi_{i}(\mathbf{R})=M$ for all $\mathbf{R} \in \mathcal{V}^{n}$.

Rules require each agent to report a preference. A rule is strategy-proof if it is always in the best interest of an agent to reveal his preferences truthfully. Formally,

Definition 1 A rule on $\mathcal{V}^{n}$, $\Phi$, is strategy-proof if for all $\mathbf{R}=\left(R_{1}, \ldots, R_{n}\right) \in \mathcal{V}^{n}$, all $i \in N$, and all $R \in \mathcal{V}$, we have $\Phi_{i}\left(R_{i}, \mathbf{R}_{-i}\right) R_{i} \Phi_{i}\left(R, \mathbf{R}_{-i}\right)$.

Given a preference profile $\mathbf{R} \in \mathcal{V}^{n}$, an allocation $x \in Z$ is efficient if there is no $z \in Z$ such that for all $i \in N, z_{i} R_{i} x_{i}$, and for at least one $j \in N$ we have $z_{j} P_{j} x_{j}$. Denote by $E(\mathbf{R})$ the set of efficient allocations. A rule is efficient if it selects an efficient allocation. Formally,

Definition 2 A rule on $\mathcal{V}^{n}$, $\Phi$, is efficient if for all $\mathbf{R} \in \mathcal{V}^{n}$, we have $\Phi(\mathbf{R}) \in E(\mathbf{R})$. 
Here, we are specially interested in simple rules satisfying the following two properties. The first one says that the rule has the informationally nice feature that it only requires to each agent to reveal his set of best-shares since it depends only on their top-sets. This is a very common feature of all rules used in the study of the division problem. In fact, except Massó and Neme (2001) all papers in this literature have confined to study exclusively tops-only rules, although (a) tops-onlyness is not explicitly imposed on the rule but derived as a consequence of other properties (strategy-proofness and efficiency) and (b) the rule is supposed to operate on a fixed domain of preferences (single-peaked or single-plateaued ones). However, as our result in Massó and Neme (2001) points out, when the domain of the rules is not fixed (rather, the objective is to identify their maximal admissible domain) non tops-only rules arise because they may still be strategy-proof and efficient on these domains. Therefore, and again because we find it to be the most unquestionable property if we want to restrict ourselves to use simple rules, here we do explicitly require tops-onlyness but we dispense the rule to satisfy any anonymity like condition. Formally,

Definition 3 A rule on $\mathcal{V}^{n}$, $\Phi$, is tops-only if for all $\mathbf{R}, \mathbf{R}^{\prime} \in \mathcal{V}^{n}$ such that $t\left(R_{i}\right)=$ $t\left(R_{i}^{\prime}\right)$ for all $i \in N$, we have $\Phi(\mathbf{R})=\Phi\left(\mathbf{R}^{\prime}\right)$.

Therefore, a tops-only rule $\Phi$ can be seen as a mapping $\Phi: \mathcal{T}^{n} \rightarrow Z$, where $\mathcal{T}$ is the family of all non-empty and closed subsets of $[0, M]$. Since best-shares sets will be closed intervals (Proposition 1 will establish this fact) we define $\mathcal{I}=\{[a, b] \subseteq$ $[0, M] \mid 0 \leq a \leq b \leq M\}$ as the family of all closed intervals contained in $[0, M]$.

The second property related to simple rules refers to a weak requirement of continuity.

Definition 4 A tops-only rule on $\mathcal{V}^{n}, \Phi$, is continuous if it is continuous with the Hausdorff topology. ${ }^{5}$

Following Ching and Serizawa (1998) we can define, given a list of properties that a rule may satisfy, the concept of "a maximal domain of preferences for this list".

Definition $5 A$ set $\mathcal{R}_{m}$ of preferences is a maximal domain for a list of properties if: (1) $\mathcal{R}_{m} \subseteq \mathcal{R}$; (2) there exists a rule on $\mathcal{R}_{m}^{n}$ satisfying the properties; and (3) there is no rule on $\mathcal{Q}^{n}$ satisfying the same properties such that $\mathcal{R}_{m} \subsetneq \mathcal{Q} \subseteq \mathcal{R}$.

\footnotetext{
${ }^{5}$ The Hausdorff topology on $\mathcal{V}^{n}$ is the induced topology from the Hausdorff topology on $\mathcal{T}^{n}$. See page 16 in Hildenbrand (1974) for the definition of the Hausdorff distance between sets.
} 


\section{Results}

Our main result identifies a maximal domain of preference relations, closely related to the set of single-plateaued preferences, admitting strategy-proof, efficient, topsonly, and continuous rules. ${ }^{6}$ This set, which depends on $M$ and $n$, is strictly larger than the set of single-plateaued preferences. In order to provide the reader with some intuitions about the exact shape of preference relations in this maximal domain, we will obtain it in several steps.

\subsection{Single-plateaued Preferences}

A preference is said to be single-plateaued if the set of best-shares is an interval and at each of its sides the preference is strictly monotonic. Formally,

Definition 6 A preference relation $R \in \mathcal{R}$ is single-plateaued if:

(a) $t(R)=[\underline{t}(R), \bar{t}(R)]$.

(b) Given $x<y \leq \underline{t}(R)$, then $y P x$.

(c) Given $\bar{t}(R) \leq y<x$, then $y P x$.

Let $\mathcal{R}_{s p}$ be the set of single-plateaued preferences. The subset of single-plateaued preferences whose top is a singleton is called the set of single-peaked preferences and it will be denoted by $\mathcal{R}_{s}$.

The following rule on $\mathcal{V}^{n}$ constitutes a natural extension of the uniform rule (defined on the set of single-peaked preferences) to a generic domain of preferences. Ching and Serizawa (1998) used this rule to establish their maximal domain result in the context of a variable $M$.

Definition 7 The extended uniform rule on $\mathcal{V}^{n}, U$, is defined as follows: for all $\mathbf{R} \in \mathcal{V}^{n}$ and all $i \in N$,

$$
U_{i}(\mathbf{R})= \begin{cases}\min \left\{\underline{t}\left(R_{i}\right), \lambda(\mathbf{R})\right\} & \text { if } M \leq \sum \underline{t}\left(R_{j}\right), \\ \min \left\{\bar{t}\left(R_{i}\right), \underline{t}\left(R_{i}\right)+\lambda(\mathbf{R})\right\} & \text { if } \sum \underline{t}\left(R_{j}\right) \leq M \leq \sum \bar{t}\left(R_{j}\right), \\ \max \left\{\bar{t}\left(R_{i}\right), \lambda(\mathbf{R})\right\} & \text { if } \sum \bar{t}\left(R_{j}\right) \leq M,\end{cases}
$$

where $\lambda(\mathbf{R})$ solves $\sum U_{j}(\mathbf{R})=M$.

\footnotetext{
${ }^{6}$ See Moulin (1984) and Berga (1998) for characterizations of strategy-proof rules under the domain of single-plateaued preferences in a public-good context.
} 
Observe that the extended uniform rule is strategy-proof, efficient, tops-only, and continuous on the domain of single-plateaued preferences. Therefore, assume that the maximal domain of preferences under which we can design a rule with these properties contains the domain of single-plateaued preferences. Under these circumstances, we are able to establish a general property that all these preferences in this maximal domain must satisfy. Proposition 1 below states that the set of maximal shares of these preferences are closed intervals. Remark 1 says that, as a consequence of Proposition 1, efficient, tops-only, and continuous rules ration all agents in the same side of the plateau.

Proposition 1 Assume $\mathcal{R}_{s p} \subset \mathcal{V}$ and let $\Phi: \mathcal{V}^{n} \rightarrow Z$ be an efficient, tops-only, and continuous rule. Then, $t(R)=[\underline{t}(R), \bar{t}(R)]$ for all $R \in \mathcal{V}$.

Proof. See the Appendix.

Denote by $\mathcal{R}_{p}$ the set of preference relations whose sets of maximal shares are closed intervals; namely,

$$
\mathcal{R}_{p}=\{R \in \mathcal{R} \mid t(R)=[\underline{t}(R), \bar{t}(R)]\} .
$$

Observe that the maximal domain of preference relations that we are looking after has to be a subset of $\mathcal{R}_{p}$.

Remark 1 Assume $\mathcal{R}_{s p} \subset \mathcal{V}$ and let $\Phi: \mathcal{V}^{n} \rightarrow Z$ be an efficient, tops-only, and continuous rule. Then, for all $\mathbf{R} \in \mathcal{V}^{n}$ and all $i \in N$ :

(a) If $\sum \bar{t}\left(R_{j}\right) \leq M$ then $\bar{t}\left(R_{i}\right) \leq \Phi_{i}(\mathbf{R})$.

(b) If $M \leq \sum \underline{t}\left(R_{j}\right)$ then $\Phi_{i}(\mathbf{R}) \leq \underline{t}\left(R_{i}\right)$.

By Proposition 1, and abusing notation, we can look at any strategy-proof, efficient, tops-only, and continuous rule $\Phi$ on $\mathcal{V}^{n}$ as just a function $\Phi: \mathcal{I}^{n} \rightarrow Z$ defined on vectors of $n$ intervals.

\subsection{Weakly Single-plateaued Preferences}

The set of single-plateaued preferences is not a maximal domain of preferences (for our list of properties) since the extended uniform rule is strategy-proof, efficient, tops-only, and continuous on a larger domain. This domain also admits non-monotonic preferences on shares above $\frac{M}{n}$ when $\bar{t}(R) \leq \frac{M}{n}$ and below $\frac{M}{n}$ when $\frac{M}{n} \leq \underline{t}(R)$. 
Definition 8 A preference relation $R \in \mathcal{R}$ is weakly single-plateaued if:

(a) $t(R)=[\underline{t}(R), \bar{t}(R)]$.

(b) Given $x<y \leq \underline{t}(R)$ such that $\frac{M}{n} \leq y$, then $y P x$.

(c) Given $\bar{t}(R) \leq y<x$ such that $y \leq \frac{M}{n}$, then $y P x$.

Let $\mathcal{R}_{w s p}(n)$ be the set of weakly single-plateaued preferences (observe that this set depends on $n$ ).

Remark 2 The extended uniform rule $U$ is strategy-proof, efficient, tops-only, and continuous on the domain of weakly single-plateaued preferences.

Therefore, assume that the maximal domain contains the set of weakly singleplateaued preferences and it is contained in the set $\mathcal{R}_{p}$. Now, we are also able to establish very useful properties of any rule (satisfying our list of properties) on any domain containing the set $\mathcal{R}_{w s p}(n)$.

Proposition 2 Assume $\mathcal{R}_{w s p}(n) \subset \mathcal{V} \subset \mathcal{R}_{p}$ and let $\Phi: \mathcal{V}^{n} \rightarrow Z$ be a strategy-proof, efficient, tops-only, and continuous rule.

(a) Let $\left(x_{1}, \ldots, x_{n}\right)$ be a vector such that $x_{i} \leq \frac{M}{n}$ for all $i$. Then, $\Phi_{i}\left(x_{1}, \ldots, x_{n}\right)=\frac{M}{n}$ for all $i$.

(b) Let $\left(x_{1}, \ldots, x_{n}\right)$ be a vector such that $\sum x_{j} \leq M$ and assume that $x_{i} \geq \frac{M}{n}$ and $x_{j} \leq \frac{M}{n}$ for every $j \neq i$. Then $\Phi_{i}\left(x_{1}, \ldots, x_{n}\right)=x_{i}$.

(c) Let $\left(x_{1}, \ldots, x_{n}\right)$ be a vector such that $x_{i} \geq \frac{M}{n}$ for all $i$. Then, $\Phi_{i}\left(x_{1}, \ldots, x_{n}\right)=\frac{M}{n}$ for all $i$.

(d) Let $\left(x_{1}, \ldots, x_{n}\right)$ be a vector such that $\sum x_{j} \geq M$ and assume that $x_{i} \leq \frac{M}{n}$ and $x_{j} \geq \frac{M}{n}$ for every $j \neq i$. Then $\Phi_{i}\left(x_{1}, \ldots, x_{n}\right)=x_{i}$.

Proof. See the Appendix.

\subsection{Partially Single-plateaued Preferences}

Assume that the maximal domain $\mathcal{V}$ contains the set of weakly single-plateaued preferences $\mathcal{R}_{w s p}(n)$, it is contained in $\mathcal{R}_{p}$, and let $\Phi: \mathcal{V}^{n} \rightarrow Z$ be a strategy-proof, efficient, tops-only, and continuous rule. But the set of weakly single-plateaued preferences $\mathcal{R}_{w s p}(n)$ is not maximal yet. In particular, parts (a) and (b) of Proposition 3 below say that all preferences in the maximal domain satisfy a weak monotonicity property on the interval between $\frac{M}{n}$ and the set of maximal shares (observe that 
parts (b) and (c) in Definition 8 require that, for a weakly single-plateaued preference, this monotonicity property holds strictly). Moreover, parts (c) and (d) of Proposition 3 say that $\frac{M}{n}$ is strictly preferred to all shares lying further away from the plateau than $\frac{M}{n}$. Formally,

Proposition 3 Assume $\mathcal{R}_{w s p}(n) \subset \mathcal{V}$. Let $\Phi: \mathcal{V}^{n} \rightarrow Z$ be a strategy-proof, efficient, tops-only, and continuous rule and let $R \in \mathcal{V}$.

(a) Assume that $x$ and $y$ are such that $\frac{M}{n} \leq x<y \leq \underline{t}(R)$. Then, $y R x$.

(b) Assume that $x$ and $y$ are such that $\bar{t}(R) \leq y<x \leq \frac{M}{n}$. Then, $y R x$.

(c) Assume that $x$ is such that $x<\frac{M}{n}<\underline{t}(R)$. Then, $\frac{M}{n} P x$.

(d) Assume that $x$ is such that $\bar{t}(R)<\frac{M}{n}<x$. Then, $\frac{M}{n} P x$.

Proof. See the Appendix.

Let $\mathcal{R}_{m p}(n) \subset \mathcal{R}_{p}$ be the set of preference relations satisfying conditions (a), (b), (c), and (d) of Proposition 3. Observe now that the maximal domain of preferences $\mathcal{V}$ that we want to identify has the property that $\mathcal{R}_{w s p}(n) \subset \mathcal{V} \subset \mathcal{R}_{m p}(n) \subset \mathcal{R}_{p}$.

Finally, we want to illustrate why our domain admits preference relations with a very special kind of indifference intervals away from the top. Intuitively, most of these intervals are excluded because our properties impose on the rule the following two features. First, and as a consequence of the efficiency of the rule, an agent can not receive a share on an indifference interval away from the top while another agent is rationed; otherwise, both agents could be strictly better off by a transfer of some amount of the good. Second, and as a consequence of Proposition 2, it is indeed possible that such situation exists if the indifference interval is below $\frac{M}{2}$. Therefore, preferences with such indifference intervals are excluded. Examples 1 and 2 illustrate why these two features exclude some preferences while Example 3 exhibits a preference relation which is not excluded by them. In particular, preferences with indifference intervals between $\frac{M}{n}$ and $\frac{M}{2}(\leq \underline{t}(R))$ and between $\bar{t}(R)$ and $\frac{M}{2}$ (whenever $n=2)$ are excluded while preferences with indifference intervals between $\frac{M}{2}$ and $\underline{t}(R)$ are included.

Example 1 Let $M=10$ and $N=\{1,2,3\}$. Assume $\mathcal{R}_{w s p}(3) \subset \mathcal{V}$ and let $\Phi$ be a strategy-proof, efficient, tops-only, and continuous rule on $\mathcal{V}^{3}$. Consider the preference profile $\mathbf{R}=\left(R_{1}, R_{2}, R_{3}\right)$ where, for $i=1,2$,

$$
y P_{i} x \text { for all } 0 \leq x<y \leq 10
$$


and

$$
y P_{3} x \text { for all } 0 \leq x<y \leq 3 \text { and all } 4 \leq x<y \leq 10
$$

and

$$
y I_{3} x \text { for all } x, y \in[3,4] \text {. }
$$

By Proposition 2, part (c), $\Phi(10,10,10)=\left(\frac{10}{3}, \frac{10}{3}, \frac{10}{3}\right)$. Since $\Phi$ is tops-only, and assuming that $R_{3} \in \mathcal{V}, \Phi\left(R_{1}, R_{2}, R_{3}\right)=\left(\frac{10}{3}, \frac{10}{3}, \frac{10}{3}\right)$. The vector $(3.5,3.5,3)$ is feasible and $3.5 P_{i} \Phi_{i}\left(R_{1}, R_{2}, R_{3}\right)$ for $i=1,2$ and $3 I_{3} \Phi_{3}\left(R_{1}, R_{2}, R_{3}\right)$. Therefore, $\Phi\left(R_{1}, R_{2}, R_{3}\right) \notin E\left(R_{1}, R_{2}, R_{3}\right)$, which contradicts the efficiency of $\Phi$. Hence, $R_{3}$ can not belong to $\mathcal{V}$.

Example 2 Let $M=10$ and $N=\{1,2\}$. Assume $\mathcal{R}_{w s p}(2) \subset \mathcal{V}$ and let $\Phi$ is a strategy-proof, efficient, tops-only, and continuous rule on $\mathcal{V}^{2}$. Consider the preference profile $\mathbf{R}=\left(R_{1}, R_{2}\right)$, where,

$$
\begin{gathered}
\qquad y P_{1} x \text { for all } 0 \leq y<x \leq 10 \\
y P_{2} x \text { for all } 0 \leq y<x \leq 3 \text { and all } 7 \leq y<x \leq 10
\end{gathered}
$$

and

$$
y I_{2} x \text { for all } x, y \in[3,7] \text {. }
$$

By Proposition 2, part (a), $\Phi(0,0)=(5,5)$. Since $\Phi$ is tops-only, and assuming that $R_{2} \in \mathcal{V}, \Phi\left(R_{1}, R_{2}\right)=(5,5)$. The vector $(3,7)$ is feasible and $3 P_{1} \Phi_{1}\left(R_{1}, R_{2}\right)$ and $7 I_{2} \Phi_{2}\left(R_{1}, R_{2}\right)$. Therefore, $\Phi\left(R_{1}, R_{2}\right) \notin E\left(R_{1}, R_{2}\right)$, which contradicts the efficiency of $\Phi$. Hence, $R_{2}$ can not belong to $\mathcal{V}$.

Example 3 Let $M=10$ and $N=\{1,2\}$. Assume $\mathcal{R}_{w s p}(2) \subset \mathcal{V}$ and consider the extended uniform rule $U$ on $\mathcal{V}^{2}$. Take the preference relation $R_{1}$, where

$$
x P_{1} y \text { for all } 0 \leq y<x \leq 6 \text { and all } 7 \leq y<x \leq 10
$$

and

$$
y I_{1} x \text { for all } x, y \in[6,7] \text {. }
$$

Let $R_{2} \in \mathcal{V}$ be any preference relation such that $U_{1}\left(R_{1}, R_{2}\right) \in(6,7)$. We want to show that $U_{2}\left(R_{1}, R_{2}\right) \in t\left(R_{2}\right)$. To see it, observe that $U_{1}\left(R_{1}, R_{2}\right)<\underline{t}\left(R_{1}\right)$ implies, by part (b) of Remark $1, U_{2}\left(R_{1}, R_{2}\right) \leq \underline{t}\left(R_{2}\right)$. To obtain a contradiction, assume $U_{2}\left(R_{1}, R_{2}\right)<\underline{t}\left(R_{2}\right)$. Take any $\bar{R}_{2} \in \mathcal{R}_{s p}$ with the properties that $t\left(\bar{R}_{2}\right)=t\left(R_{2}\right)$ and $5 \bar{P}_{2} U_{2}\left(R_{1}, R_{2}\right)$. Since $U(10,10)=(5,5)$ and $U$ is tops-only, $U\left(R_{1}, \widehat{R}_{2}\right)=(5,5)$ 
whenever $t\left(\widehat{R}_{2}\right)=10$. But then, agent 2 manipulates $U$ at $\left(R_{1}, \bar{R}_{2}\right)$ by declaring any $\widehat{R}_{2}$ with $t\left(\widehat{R}_{2}\right)=10$.

Before stating the formal definition, and as a way of recapitulating some of the previous results, it seems useful to give a verbal explanation of the set of partially single-plateaued preferences. A preference relation $R \in \mathcal{R}$ is partially singleplateaued if its set of best shares is a closed interval and the following additional properties are satisfied: (a) If $\frac{M}{n}<\underline{t}(R)$, then the preference has to be "increasing" between $M / n$ and its smallest best share $\underline{t}(R)$, although it may have intervals of indifference provided these intervals are above $M / 2$. Moreover, the egalitarian share $M / n$ has to be strictly preferred to all smaller shares, but all orderings are possible among them. (b) If $\bar{t}(R)<\frac{M}{n}$, then the preference has to be "decreasing" between its largest best share $\bar{t}(R)$ and $M / n$, although it may have intervals of indifference provided that $n=2$ and these intervals are below $M / 2$. Moreover, the egalitarian share $M / n$ has to be strictly preferred to all larger shares, but also all orderings are possible among them. Finally, if $\underline{t}(R) \leq M / n \leq \bar{t}(R)$, then, no additional requirement is imposed..$^{7}$ Formally,

Definition 9 A preference relation $R \in \mathcal{R}$ is partially single-plateaued if:

(a) $t(R)=[\underline{t}(R), \bar{t}(R)]$.

(b) Given $x<y \leq \underline{t}(R)$ such that $\frac{M}{n} \leq y$, then:

(b.1) $y R x$, and

(b.2) if $x$ Iy then $\frac{M}{2} \leq x<y<\underline{t}(R)$.

(c) Given $\bar{t}(R) \leq y<x$ such that $y \leq \frac{M}{n}$, then:

(c.1) $y R x$, and

(c.2) if $x$ Iy then $n=2$ and $x \leq \frac{M}{n}$.

We denote by $\mathcal{R}_{p s p}(n)$ the set of partially single-plateaued preferences. Note that $\mathcal{R}_{w s p}(n) \subsetneq \mathcal{R}_{p s p}(n) \subsetneq \mathcal{R}_{m p}(n)$ for all $n \geq 2$. Figure 1 illustrates three possible types of partially single-plateaued preferences depending on whether $\frac{M}{n} \in[\underline{t}(R), \bar{t}(R)]$, $\frac{M}{n}<\underline{t}(R)$ (as well as $\left.\frac{M}{2}<\underline{t}(R)\right)$, and $\bar{t}(R)<\frac{M}{n}$.

${ }^{7}$ The set of feebly single-plateaued preferences identified in Massó and Neme (2001) satisfy the same properties but in (a) the intervals of indifference have to be sufficiently large in relation to $M$ (the sum of the extremes has to be larger than $M$ ), although they do not have to be necessarily above $M / 2$; in (b) the intervals of indifference have to be sufficiently small in relation to $M$ (the sum of the extremes has to be smaller than $M$ ) although no condition on $n$ is imposed and the intervals of indifference do not have to be necessarily below $M / 2$; and moreover $M / n$, instead of strictly preferred, has to be at least as good as all smaller shares (in (a)) and all larger shares (in (b)). 
Insert Figure 1 about here

Figure 2 illustrates a preference relation with an indifference interval below $M / 2$ that is partially single-plateaued for $n=2$ and $n=4$ but it is not for $n=3$.

Insert Figure 2 about here

Now, we can state our result.

Theorem 1 The set of partially single-plateaued preferences, $\mathcal{R}_{p s p}(n)$, is a maximal domain for the properties of strategy-proofness, efficiency, tops-onlyness, and continuity.

Proof. See the Appendix.

Figure 3 illustrates the process used to identify the set of partially single-plateaued preferences as the maximal domain of preferences for the properties of strategyproofness, efficiency, tops-onlyness, and continuity.

Insert Figure 3 about here

\section{Final Remarks}

We close with five remarks. First, the reader should not be surprised by the fact that, on the one hand, a rule responds only to the information about the top ranking shares of preferences and, on the other hand, the maximal domain of preferences crucially depends on the information about other non-top ranking shares. Strategy-proofness and efficiency exclude only some preferences while others are admitted. To better understand that this is not contradictory consider the uniform rule defined on any domain of preferences having a unique top; it is a tops-only rule, and strategy-proof and efficient on the domain of single-peaked preferences, as well. Yet, if the domain of single-peaked preferences is enlarged by admitting a non-monotonic ordering (at one side of the top) then the uniform rule fails to be either strategy-proof or efficient (or both). That is, the rule does not pay attention to information other than the top ranked shares and yet its strategy-proofness and efficiency depend on whether or not the domain admits preferences which order in a particular way non-top ranked shares. 
Second, parts (a) and (c) of Proposition 2 suggest that the properties of strategyproofness, efficiency, tops-onlyness, and continuity imply (surprisingly) some partial anonymity condition. But this is only apparent. It is a consequence of the following two unrelated reasons: (i) An indirect anonymity axiom is assumed when we impose the natural condition that the domain of the rule be the Cartesian product of the same set of preferences for all agents. Otherwise, given an arbitrary feasible vector $\left(y_{1}, \ldots, y_{n}\right)$, we could define agent $i$ 's specific maximal set of preferences by replacing in Definition 9 the share $\frac{M}{n}$ by $y_{i}$. Then, the statement of Proposition 2 would also hold after replacing the role of the vector of equal shares $\left(\frac{M}{n}, \ldots, \frac{M}{n}\right)$ by $\left(y_{1}, \ldots, y_{n}\right)$. (ii) We are looking for a maximal domain of preferences. There are other domains of preferences (for instance, the set of preferences satisfying Definition 9 after replacing $\frac{M}{n}$ by $\frac{M}{2 n}$ in the hypothesis to require condition (b.1)) under which we could have a vector $\left(x_{1}, \ldots, x_{n}\right)$ with the property that $x_{i} \geq \frac{M}{n}$ for all $i$, but $\frac{M}{2 n} \leq \Phi_{j}\left(x_{1}, \ldots, x_{n}\right)<$ $\frac{M}{n}$ for some $j$; hence, $\Phi$ does not satisfy the partial anonymity condition of part (c) of Proposition 2. However, this domain of preferences would not be maximal since it is smaller than $\mathcal{R}_{p s p}(n)$ because these preferences are all strictly monotonic between $\frac{M}{2 n}$ and $\frac{M}{n},{ }^{8}$ while preferences in $\mathcal{R}_{p s p}(n)$ do not have to. This lack of anonymity (or symmetry) suggests, though, the possibility that our list of properties admits, for the domain of partially single-plateaued preferences, a large class of functions (and not only the extended uniform rule) satisfying our list of properties. ${ }^{9}$

Third, the maximal domain of preferences identified in Ching and Serizawa (1998) and Massó and Neme (2001) is, in each case, the unique one containing the set of single-peaked preferences. In contrast (and as another consequence of not requiring any property related to symmetry or anonymity), the set of partially single-plateaued preferences is not the unique one containing the set of single-peaked preferences for our list of properties. To see it, consider the domain of preferences $\mathcal{R}_{L} \subseteq \mathcal{R}$ where $R_{i} \in \mathcal{R}_{L}$ if and only if:

$$
y P_{i} x \text { for all } 0 \leq x<y \leq \underline{t}\left(R_{i}\right)
$$

Let $N=\{1,2,3\}$ and consider the rule $\psi$ on $\left(\mathcal{R}_{L}\right)^{3}$ defined as follows: for all $\mathbf{R} \in\left(\mathcal{R}_{L}\right)^{3}$,

$$
\psi_{1}(\mathbf{R})=\underline{t}\left(R_{1}\right)
$$

\footnotetext{
${ }^{8}$ The same argument would apply if we replace $\frac{M}{n}$ in Definition 9 by any smaller share.

${ }^{9}$ Barberà, Jackson, and Neme (1997) shows that, on the single-peaked domain, the class is larger even if we add replacement monotonicity to our list of properties.
} 


$$
\begin{aligned}
& \psi_{2}(\mathbf{R})=\max \left\{M-\underline{t}\left(R_{1}\right), \underline{t}\left(R_{2}\right)\right\}, \text { and } \\
& \psi_{3}(\mathbf{R})=M-\psi_{1}(\mathbf{R})-\psi_{2}(\mathbf{R}) .
\end{aligned}
$$

It is easy to check that $\psi:\left(\mathcal{R}_{L}\right)^{3} \rightarrow Z$ is strategy-proof, efficient, tops-only, and continuous. Moreover, it is possible to show that $\mathcal{R}_{L}$ is a maximal domain of preferences for this list of properties.

Fourth, the intersection of all our maximal domains of preferences, fixed $M$ and when $n$ varies from two to infinity, is strictly larger than the set of single-plateaued preferences; namely, $\bigcap_{n \geq 2} \mathcal{R}_{p s p}(n) \supsetneq \mathcal{R}_{s p}$. To see that, observe that there are many preference relations that are partially single-plateaued for all $n \geq 2$ but they are not single-plateaued.

Fifth, the intersection of all our maximal domains of preferences, fixed $n \geq 2$ and when $M$ varies from zero to infinity, coincides with the single-plateaued domain. This implies that, when the rule depends not only on preferences but also on the amount $M$ to be allocated (as in Ching and Serizawa, 1998), the maximal domain coincides with the set of single-plateaued preferences as already shown by Ching and Serizawa (1998) for the properties of strategy-proofness, efficiency, and symmetry.

\section{Appendix}

\subsection{Proofs of Propositions 1 and 2}

To prove Propositions 1 and 2 we need the following notation. Given $[a, b] \subseteq[0, M]$, let $R^{[a, b]}$ be any preference relation in $\mathcal{R}_{s p}$ with the property that $t\left(R^{[a, b]}\right)=[a, b]$. Again, to stress the role of agent $i$ 's preference relation (or the preference relations of agents in $S$ ) we will represent a profile of preferences, given a vector $[\mathbf{a}, \mathbf{b}]=$ $\left(\left[a_{1}, b_{1}\right], \ldots,\left[a_{n}, b_{n}\right]\right)$, as $\mathbf{R}^{[\mathbf{a}, \mathbf{b}]} \equiv\left(R_{i}^{\left[a_{i}, b_{i}\right]}, \mathbf{R}_{-i}^{[\mathbf{a}, \mathbf{b}]}\right)=\left(\mathbf{R}_{S}^{\left[\mathbf{a}_{S}, \mathbf{b}_{S}\right]}, \mathbf{R}_{-S}^{[\mathbf{a}, \mathbf{b}]}\right)$. Finally, given $[a, b] \subseteq[0, M]$, we will represent by $\mathbf{R}^{[a, b]}$ any preference profile $\left(R_{1}^{[a, b]}, \ldots, R_{n}^{[a, b]}\right)$.

Proof of Proposition 1 Assume otherwise; that is, there exist $R \in \mathcal{V}$ and $y \in$ $(\underline{t}(R), \bar{t}(R))$ such that $\underline{t}(R) P y$. Define

$$
\underline{x}=\frac{M-\underline{t}(R)}{n-1}
$$

and

$$
\bar{x}=\frac{M-\bar{t}(R)}{n-1}
$$


Let $i$ be arbitrary. Since $\Phi$ is efficient,

$$
\Phi_{j}\left(R, \mathbf{R}_{-i}^{\underline{x}}\right)= \begin{cases}\underline{t}(R) & \text { if } j=i \\ \underline{x} & \text { if } j \neq i\end{cases}
$$

and

$$
\Phi_{j}\left(R, \mathbf{R}_{-i}^{\bar{x}}\right)=\left\{\begin{array}{ll}
\bar{t}(R) & \text { if } j=i \\
\bar{x} & \text { if } j \neq i
\end{array} .\right.
$$

It is easy to show that there exists $z \in[\bar{x}, \underline{x}]$ such that either $\Phi_{i}\left(R, \mathbf{R}_{-i}^{[z, \underline{x}]}\right)>y$ or $\Phi_{i}\left(R, \mathbf{R}_{-i}^{[\bar{x}, z]}\right)<y$. Without loss of generality, assume there exists $z \in[\bar{x}, \underline{x}]$ such that $\Phi_{i}\left(R, \mathbf{R}_{-i}^{[z, \underline{x}]}\right)>y$. By continuity of $\Phi$ there exists $z^{\prime} \in[z, \underline{x}]$ such that $\Phi_{i}\left(R, \mathbf{R}_{-i}^{\left[z^{\prime}, \underline{x}\right]}\right)=y$. But the vector $\left(\alpha_{1}, \ldots, \alpha_{n}\right)$, where

$$
\alpha_{j}= \begin{cases}\underline{t}(R) & \text { if } j=i \\ \underline{x} & \text { if } j \neq i\end{cases}
$$

is feasible and has the property that $\alpha_{i} P y$ and $\alpha_{j} R_{j}^{\left[z^{\prime}, \underline{x}\right]} \Phi_{j}\left(R, \mathbf{R}_{-i}^{\left[z^{\prime}, \underline{x}\right]}\right)$ for all $j \neq i$. Therefore, $\Phi\left(R, \mathbf{R}_{-i}^{\left[z^{\prime}, x\right]}\right) \notin E\left(R, \mathbf{R}_{-i}^{\left[z^{\prime}, x\right]}\right)$, contradicting the efficiency of $\Phi$.

Proof of Proposition 2 (a) Suppose otherwise; that is, there exist $\mathbf{x}=\left(x_{1}, \ldots, x_{n}\right) \in$ $[0, M]^{n}$ and $i \in N$ with the properties that $x_{j} \leq \frac{M}{n}$ for all $j$ and $\Phi_{i}\left(x_{1}, \ldots, x_{n}\right) \neq$ $\frac{M}{n}$. By Remark 1, part (a), we may assume (since $\Phi\left(x_{1}, \ldots x_{n}\right) \in Z$ ) that $\frac{M}{n}<$ $\Phi_{i}\left(x_{1}, \ldots, x_{n}\right) \leq X$ holds, where $X=M-\sum_{j \neq i} x_{j}$. Without loss of generality assume $i \neq 1$. By efficiency of $\Phi, \Phi\left(R_{1}^{\left[x_{1}, X\right]}, \mathbf{R}_{-1}^{\mathbf{x}}\right)=\left(X, x_{2}, \ldots, x_{n}\right)$. Since $\Phi_{i}\left(R_{1}^{\left[x_{1}, X\right]}, \mathbf{R}_{-1}^{\mathbf{x}}\right)=x_{i} \leq \frac{M}{n}<\Phi_{i}\left(R_{1}^{x_{1}}, \mathbf{R}_{-1}^{\mathbf{x}}\right) \leq X$. By continuity of $\Phi$, for every $r \in\left(\frac{M}{n}, \Phi_{i}\left(R_{1}^{x_{1}}, \mathbf{R}_{-1}^{\mathbf{x}}\right)\right)$ there exists $y \in\left[x_{1}, X\right]$ such that $\Phi_{i}\left(R_{1}^{\left[x_{1}, y\right]}, \mathbf{R}_{-1}^{\mathbf{x}}\right)=r$. Then, by efficiency of $\Phi$,

$$
\Phi_{i}\left(R_{1}^{\left[x_{1}, y\right]}, R_{i}^{X}, \mathbf{R}_{-\{1, i\}}^{\mathbf{x}}\right)=X
$$

for any $R_{i}^{X} \in \mathcal{R}_{w s p}(n)$. Consider now any preference $R_{i}^{\prime} \in \mathcal{R}_{w s p}(n)$ with the properties that $t\left(R_{i}^{\prime}\right)=x_{i}$ and

$$
X P_{i}^{\prime} r
$$

Since $\Phi$ is tops-only,

$$
\Phi_{i}\left(R_{1}^{\left[x_{1}, y\right]}, R_{i}^{x_{i}}, \mathbf{R}_{-\{1, i\}}^{\mathbf{x}}\right)=r=\Phi_{i}\left(R_{1}^{\left[x_{1}, y\right]}, R_{i}^{\prime}, \mathbf{R}_{-\{1, i\}}^{\mathbf{x}}\right) .
$$


But then, conditions (1), (2), and (3) imply

$$
\Phi_{i}\left(R_{1}^{\left[x_{1}, y\right]}, R_{i}^{X}, \mathbf{R}_{-\{1, i\}}^{\mathbf{x}}\right) P_{i}^{\prime} \Phi_{i}\left(R_{1}^{\left[x_{1}, y\right]}, R_{i}^{\prime}, \mathbf{R}_{-\{1, i\}}^{\mathbf{x}}\right),
$$

which means that $\Phi$ is not strategy-proof.

(b) Let $\mathbf{x}=\left(x_{1}, \ldots, x_{n}\right)$ be a vector such that $\sum x_{j} \leq M, x_{i} \geq \frac{M}{n}$ for $i$, and $x_{j} \leq$ $\frac{M}{n}$ for all $j \neq i$. By efficiency of $\Phi$, we may assume that $\Phi_{i}\left(x_{1}, \ldots, x_{n}\right) \geq x_{i}$. To get a contradiction, assume $\Phi_{i}\left(x_{1}, \ldots, x_{n}\right)>x_{i}$. Consider any preference $R^{\prime} \in \mathcal{R}_{w s p}(n)$ such that $t\left(R^{\prime}\right)=x_{i}$ and $\frac{M}{n} P^{\prime} \Phi_{i}\left(x_{1}, \ldots, x_{n}\right)$. Since $\Phi$ is tops-only,

$$
\Phi_{i}\left(R^{\prime}, \mathbf{R}_{-i}^{\mathbf{x}}\right)=\Phi_{i}\left(R^{x_{i}}, \mathbf{R}_{-i}^{\mathbf{x}}\right)
$$

On the other hand, by part (a), $\Phi_{i}\left(R^{0}, \mathbf{R}_{-i}^{\mathbf{x}}\right)=\frac{M}{n}$, which implies, together with condition (4), that $\Phi$ is not strategy-proof because

$$
\Phi_{i}\left(R^{0}, \mathbf{R}_{-i}^{\mathbf{x}}\right) P^{\prime} \Phi_{i}\left(R^{\prime}, \mathbf{R}_{-i}^{\mathbf{x}}\right)
$$

The proofs of parts (c) and (d) are omitted since they follow arguments which are symmetric to the ones used to prove parts (a) and (b), respectively.

\subsection{Proof of Proposition 3}

Proof of Proposition 3 (a) Assume otherwise; that is, there exist $x$ and $y$ such that $\frac{M}{n} \leq x<y \leq \underline{t}(R)$ and $x P y$. Consider any $R^{M} \in \mathcal{R}_{s}$. Because $\Phi_{i}\left(R^{M}, \mathbf{R}_{-i}^{0}\right)=$ $M, \Phi_{i}\left(R^{M}, \mathbf{R}_{-i}^{M / n}\right)=\frac{M}{n}$, by continuity of $\Phi$ there exists $z \leq M / n$ such that

$$
\Phi_{i}\left(R^{M}, \mathbf{R}_{-i}^{z}\right)=y
$$

By strategy-proofness of $\Phi$,

$$
\Phi_{i}\left(R, \mathbf{R}_{-i}^{z}\right) \leq y,
$$

otherwise $\Phi_{i}\left(R, \mathbf{R}_{-i}^{z}\right) P^{M} \Phi_{i}\left(R^{M}, \mathbf{R}_{-i}^{z}\right)$.

Because $z \leq \frac{M}{n}$ and Proposition 2, part (a), $\Phi_{i}\left(R^{0}, \mathbf{R}_{-i}^{z}\right)=\frac{M}{n}$. By continuity of $\Phi$ there exists $z^{\prime}$ such that $\Phi_{i}\left(R^{z^{\prime}}, \mathbf{R}_{-i}^{z}\right)=x$. By strategy-proofness of $\Phi$, $\Phi_{i}\left(R, \mathbf{R}_{-i}^{z}\right) R x P y$, which imply, by condition $(6), \Phi_{i}\left(R, \mathbf{R}_{-i}^{z}\right)<y$. Let $R^{\prime} \in \mathcal{R}_{w s p}(n)$ be such that $t\left(R^{\prime}\right)=t(R)$. Because $\Phi$ is tops-only, $\Phi_{i}\left(R^{\prime}, \mathbf{R}_{-i}^{z}\right)=\Phi_{i}\left(R, \mathbf{R}_{-i}^{z}\right)$. Therefore, by condition (5)

$$
\Phi_{i}\left(R^{M}, \mathbf{R}_{-i}^{z}\right) P^{\prime} \Phi_{i}\left(R^{\prime}, \mathbf{R}_{-i}^{z}\right),
$$

which contradicts strategy-proofness of $\Phi$. 
(c) Assume $x<\frac{M}{n}<\underline{t}(R)$ and $x R \frac{M}{n}$. By Proposition 2, part (c), $\Phi\left(R^{\underline{t}(R)}, \mathbf{R}_{-i}^{M}\right)=$ $\left(\frac{M}{n}, \ldots, \frac{M}{n}\right)$. Consider any preference profile $\left(R^{t(R)}, \mathbf{R}_{-i}^{M}\right) \in \mathcal{R}_{s}^{n}$ and any preference relation $R^{[\underline{t}(R), \bar{t}(R)]} \in \mathcal{R}_{s p}$. Since $\Phi$ is strategy-proof, $\Phi_{i}\left(R^{[\underline{\underline{t}}(R), \bar{t}(R)]}, \mathbf{R}_{-i}^{M}\right)=\frac{M}{n}$, and by tops-onlyness of $\Phi, \Phi_{i}\left(R, \mathbf{R}_{-i}^{M}\right)=\frac{M}{n}$. Now, for each $j \neq i$ there exists $\alpha_{j}>0$ such that $\sum_{j \neq i} \alpha_{j}=\frac{M}{n}-x$ and $\Phi_{j}\left(R, \mathbf{R}_{-i}^{M}\right)+\alpha_{j} \leq M$. Then, the vector of feasible shares $\left(z_{1}, \ldots, z_{n}\right)$, where

$$
z_{j}=\left\{\begin{array}{ll}
x & \text { if } j=i \\
\Phi_{j}\left(R, \mathbf{R}_{-i}^{M}\right)+\alpha_{j} & \text { if } j \neq i
\end{array},\right.
$$

has the property that $z_{i} R \Phi_{i}\left(R, \mathbf{R}_{-i}^{M}\right)$ and $z_{j} P_{j}^{M} \Phi_{j}\left(R, \mathbf{R}_{-i}^{M}\right)$ for every $j \neq i$, implying that $\Phi\left(R, \mathbf{R}_{-i}^{M}\right) \notin E\left(R, \mathbf{R}_{-i}^{M}\right)$, which contradicts the efficiency of $\Phi$.

The proof of parts (b) and (d) are omitted since they follow arguments which are symmetric to the ones used to prove parts (a) and (c), respectively.

\subsection{Proof of Theorem 1}

It is easy to check that the extended uniform rule $U$ on $\left(\mathcal{R}_{p s p}(n)\right)^{n}$ satisfies the properties of strategy-proofness, efficiency, tops-onlyness, and continuity. Assume that $\mathcal{V} \subseteq \mathcal{R}$ is a maximal domain for these properties and $\mathcal{R}_{p s p}(n) \subseteq \mathcal{V}$. We will show that $\mathcal{V}=\mathcal{R}_{p s p}(n)$, indeed.

Lemma 1 Let $R \in \mathcal{V}$. Assume that $x$ and $y$ are such that $\frac{M}{n} \leq x<y \leq$ $\min \left\{\frac{M}{2}, \underline{t}(R)\right\}$. Then, $y P x$.

Proof. First notice that the hypothesis of Lemma 1 imply that $n \geq 3$. Assume $\frac{M}{n} \leq x<y \leq \min \left\{\frac{M}{2}, \underline{t}(R)\right\}$ and $x R y$. By Proposition 3, part (a), we may assume that there exist $a$ and $b$ such that $\frac{M}{n} \leq a<b \leq \min \left\{\frac{M}{2}, \underline{t}(R)\right\}$ and $a I \widehat{x}$ for all $\widehat{x} \in[a, b]$. By efficiency of $\Phi$, for all $j \geq 3$,

$$
\Phi_{j}\left(R_{1}^{M}, R_{2}^{M}, \mathbf{R}_{-\{1,2\}}^{0}\right)=0 .
$$

Hence, there exists $i \in\{1,2\}$ such that $\Phi_{i}\left(R_{1}^{M}, R_{2}^{M}, \mathbf{R}_{-\{1,2\}}^{0}\right) \geq \frac{M}{2}$. Assume that $i=1$. Since $\Phi$ is strategy-proof,

$$
\Phi_{1}\left(R_{1}^{t(R)}, R_{2}^{M}, \mathbf{R}_{-\{1,2\}}^{0}\right) \geq \min \left\{\frac{M}{2}, \underline{t}(R)\right\} .
$$

To see it, assume $z^{\prime}=\Phi_{1}\left(R_{1}^{t(R)}, R_{2}^{M}, \mathbf{R}_{-\{1,2\}}^{0}\right)<\min \left\{\frac{M}{2}, \underline{t}(R)\right\}$ and consider any $R^{\underline{t}(R)} \in \mathcal{R}_{s}$ such that $\widehat{z} P^{\underline{t}(R)} z^{\prime}$ for all $\widehat{z} \in\left[\min \left\{\frac{M}{2}, \underline{t}(R)\right\}, M\right]$; then agent 1 
would manipulate $\Phi$ at profile $\left(R_{1}^{t(R)}, R_{2}^{M}, \mathbf{R}_{-\{1,2\}}^{0}\right)$ with $R_{1}^{M}$. Using again strategyproofness of $\Phi$, condition (7) implies

$$
\Phi_{1}\left(R_{1}^{[\underline{t}(R), \bar{t}(R)]}, R_{2}^{M}, \mathbf{R}_{-\{1,2\}}^{0}\right) \geq \min \left\{\frac{M}{2}, \underline{t}(R)\right\} .
$$

Hence, by tops-onlyness of $\Phi$,

$$
\Phi_{1}\left(R, R_{2}^{M}, \mathbf{R}_{-\{1,2\}}^{0}\right) \geq \min \left\{\frac{M}{2}, \underline{t}(R)\right\} .
$$

By Proposition 3, part (c),

$$
\Phi\left(R_{1}^{t(R)}, R_{2}^{M}, \mathbf{R}_{-\{1,2\}}^{M}\right)=\left(\frac{M}{n}, \ldots, \frac{M}{n}\right) .
$$

Since $\Phi$ is strategy-proof, $\Phi_{1}\left(R_{1}^{[t(R), \bar{t}(R)]}, R_{2}^{M}, \mathbf{R}_{-\{1,2\}}^{M}\right)=M / n$. Since $\Phi$ is tops-only, we may assume that $R^{M} \in \mathcal{R}_{s}$ and

$$
\Phi_{1}\left(R, R_{2}^{M}, \mathbf{R}_{-\{1,2\}}^{M}\right)=\frac{M}{n} .
$$

Let $x^{\prime} \in(a, b)$ be arbitrary. Conditions (8) and (9) and continuity of $\Phi$ imply that there exists $z \in[0, M]$ such that $\Phi_{1}\left(R, R_{2}^{M}, \mathbf{R}_{-\{1,2\}}^{z}\right)=x^{\prime}$; and thus, $\Phi_{2}\left(R, R_{2}^{M}, \mathbf{R}_{-\{1,2\}}^{z}\right)<M$. Therefore, there exists $\epsilon>0$ sufficiently small such that

$$
\left[\Phi_{1}\left(R, R_{2}^{M}, \mathbf{R}_{-\{1,2\}}^{M}\right)-\epsilon\right] I \Phi_{1}\left(R, R_{2}^{M}, \mathbf{R}_{-\{1,2\}}^{M}\right)
$$

and

$$
\left[\Phi_{2}\left(R, R_{2}^{M}, \mathbf{R}_{-\{1,2\}}^{M}\right)+\epsilon\right] P_{2}^{M} \Phi_{2}\left(R, R_{2}^{M}, \mathbf{R}_{-\{1,2\}}^{M}\right) .
$$

The existence of the feasible vector $\left(\alpha_{1}, \ldots, \alpha_{n}\right)$, where

$$
\begin{aligned}
\alpha_{1} & =\left[\Phi_{1}\left(R, R_{2}^{M}, \mathbf{R}_{-\{1,2\}}^{M}\right)-\epsilon\right], \\
\alpha_{2} & =\left[\Phi_{2}\left(R, R_{2}^{M}, \mathbf{R}_{-\{1,2\}}^{M}\right)+\epsilon\right], \text { and } \\
\alpha_{j} & =\Phi_{j}\left(R, R_{2}^{M}, \mathbf{R}_{-\{1,2\}}^{M}\right) \text { for all } j \geq 3
\end{aligned}
$$

and conditions (10) and (11) imply that $\Phi\left(R, R_{2}^{M}, \mathbf{R}_{-\{1,2\}}^{M}\right) \notin E\left(R, R_{2}^{M}, \mathbf{R}_{-\{1,2\}}^{M}\right)$, contradicting the efficiency of $\Phi$.

Lemma 2 Let $R \in \mathcal{V}$. Assume that $x$ and $y$ are such that $\bar{t}(R)<y<x \leq \frac{M}{n}$ and let $n \geq 3$ Then, $y P x$. 
Proof. Assume otherwise; that is, $n \geq 3$ and there exist $x$ and $y$ such that $\bar{t}(R)<y<x \leq \frac{M}{n}$ and $x R y$. By Proposition 3, part (b), we may assume that $x I y$ and there exist $a$ and $b$ such that $\bar{t}(R)<a<b \leq \frac{M}{n}$, aI $\widehat{x}$ for all $\widehat{x} \in[a, b], \widehat{z} P a$ for all $\bar{t}(R)<\widehat{x}<a$, and $b P \widehat{y}$ for all $b<\widehat{y}<\frac{M}{n}$. Consider any preference relation $R^{t(R)} \in \mathcal{R}_{s p}$. By Remark 1, part (b), $\Phi_{i}\left(R^{\bar{t}(R)}, R^{\bar{t}(R)}, \mathbf{R}_{-\{1,2\}}^{M}\right) \leq \underline{t}(R)$ for $i \in\{1,2\}$. By Proposition 2, part (a), $\Phi\left(R^{\bar{t}(R)}, R^{\bar{t}(R)}, \mathbf{R}_{-\{1,2\}}^{0}\right)=\left(\frac{M}{n}, \ldots, \frac{M}{n}\right)$. By continuity of $\Phi$ (and without loss of generality in the roles of agents 1 and 2), there exist $z, x^{\prime}$, and $y^{\prime}$ such that $\bar{t}(R)<x^{\prime} \leq y^{\prime}<\frac{M}{n}$,

$$
\Phi_{1}\left(R^{\bar{t}(R)}, R^{\bar{t}(R)}, \mathbf{R}_{-\{1,2\}}^{z}\right)=x^{\prime}
$$

and

$$
\Phi_{2}\left(R^{\bar{t}(R)}, R^{\bar{t}(R)}, \mathbf{R}_{-\{1,2\}}^{z}\right)=y^{\prime}
$$

Since $\bar{t}(R)<x^{\prime}$, Remark 1, part (a), implies that $2 \bar{t}(R)+(n-2) z<M$ and therefore,

$$
x^{\prime}+y^{\prime}+(n-2) z \leq M
$$

Since $\Phi$ is strategy-proof, $\Phi_{1}\left(R^{[\underline{t}(R), \bar{t}(R]}, R^{\bar{t}(R)}, \mathbf{R}_{-\{1,2\}}^{z}\right)=x^{\prime}$, and by tops-onlyness of $\Phi, \Phi_{1}\left(R, R^{\bar{t}(R)}, \mathbf{R}_{-\{1,2\}}^{z}\right)=x^{\prime}$. Now, if $\Phi_{2}\left(R, R^{\bar{t}(R)}, \mathbf{R}_{-\{1,2\}}^{z}\right) \neq \bar{t}(R)$ then $\Phi$ is not efficient since there exists $\epsilon$ sufficiently small (positive if $\Phi_{2}\left(R, R^{\bar{t}(R)}, \mathbf{R}_{-\{1,2\}}^{z}\right)<$ $\bar{t}(R)$ and negative otherwise) such that the feasible vector $\left(\alpha_{1}, \ldots, \alpha_{n}\right)$, where

$$
\alpha_{j}= \begin{cases}x^{\prime}-\epsilon & \text { if } j=1 \\ \Phi_{j}\left(R, R^{\bar{t}(R)}, \mathbf{R}_{-\{1,2\}}^{z}\right)+\epsilon & \text { if } j=2 \\ \boldsymbol{\Phi}_{j}\left(R, R^{\bar{t}(R)}, \mathbf{R}_{-\{1,2\}}^{z}\right) & \text { if } j \geq 3\end{cases}
$$

has the properties that $\alpha_{2} P^{\bar{t}(R)} \Phi_{2}\left(R, R^{\bar{t}(R)}, \mathbf{R}_{-\{1,2\}}^{z}\right), \alpha_{1} I \Phi_{1}\left(R, R^{\bar{t}(R)}, \mathbf{R}_{-\{1,2\}}^{z}\right)$, and $\alpha_{j} I_{j}^{z} \Phi_{j}\left(R, R^{\bar{t}(R)}, \mathbf{R}_{-\{1,2\}}^{z}\right)$ for all $j \geq 3$; thus, the rule $\Phi$ would not be efficient. If $\Phi_{2}\left(R, R^{\bar{t}(R)}, \mathbf{R}_{-\{1,2\}}^{z}\right)=\bar{t}(R)$, condition (12) implies that there exists an agent $j \geq 3$ such that $\Phi_{j}\left(R, R^{\bar{t}(R)}, \mathbf{R}_{-\{1,2\}}^{z}\right) \neq z$, in which case, using a similar argument, $\Phi$ would not be efficient. 


\section{References}

[1] Barberà, S.: "Notes on strategy-proof social choice functions", in Social Choice Re-examinated, Arrow, K., Sen, A., and Suzumura, K. editors, Mac Millan (1996). French version: "Sur les fonctions de choix non manipulables," Revue d'Économie Politique 106, 61-81 (1996).

[2] Barberà, S.: "An introduction to strategy-proof social choice functions", Social Choice and Welfare 18, 619-653 (2001).

[3] Barberà, S., Jackson, M., and Neme, A.: "Strategy-proof allotment rules", Games and Economic Behavior 18, 1-21 (1997).

[4] Barberà S., Massó, J., and Neme, A.: "Maximal domains of preferences preserving strategy-proofness for generalized median voter schemes", Social Choice and Welfare 16, 321-336 (1999).

[5] Barberà, S., Sonnenschein, H., and Zhou, L.: "Voting by committees", Econometrica 59, 595-609 (1991).

[6] Berga, D.: "Maximal domains and strategy-proofness in public good economies", Ph.D. thesis, Universitat Autònoma de Barcelona (1997).

[7] Berga, D.: "Strategy-proofness and single-plateaued preferences", Mathematical Social Sciences 35, 105-120 (1998).

[8] Berga, D.: "Single-peakedness and strategy-proofness of generalized median voter schemes", Social Choice and Welfare 19, 175-192 (2002).

[9] Berga, D. and Serizawa, S.: "Maximal domain for strategy-proof rules with one public good", Journal of Economic Theory 90, 39-61 (2000).

[10] Ching, S. and Serizawa, S.: "Maximal domain for the existence of strategy-proof rules", Journal of Economic Theory 78, 157-166 (1998).

[11] Hildenbrand, W.: Core and equilibria of a large economy. Princeton University Press. Princeton, New Jersey 1974.

[12] Massó, J. and Neme, A.: "Maximal domain of preferences in the division problem", Games and Economic Behavior 37, 367-387 (2001). 
[13] Moulin, H.: "Generalized Condorcet-winners for single-peaked and singleplateaued preferences", Social Choice and Welfare 1, 127-147 (1984).

[14] Serizawa, S.: "Power of voters and domain of preferences where voting by committees is strategy-proof", Journal of Economic Theory 67, 599-608 (1995).

[15] Sprumont, Y.: "The division problem with single-peaked preferences: a characterization of the uniform allocation rule", Econometrica 59, 509-519 (1991).

[16] Sprumont, Y.: "Strategy-proof collective choice in economic and political environments", Canadian Journal of Economics 28, 68-107 (1995). 

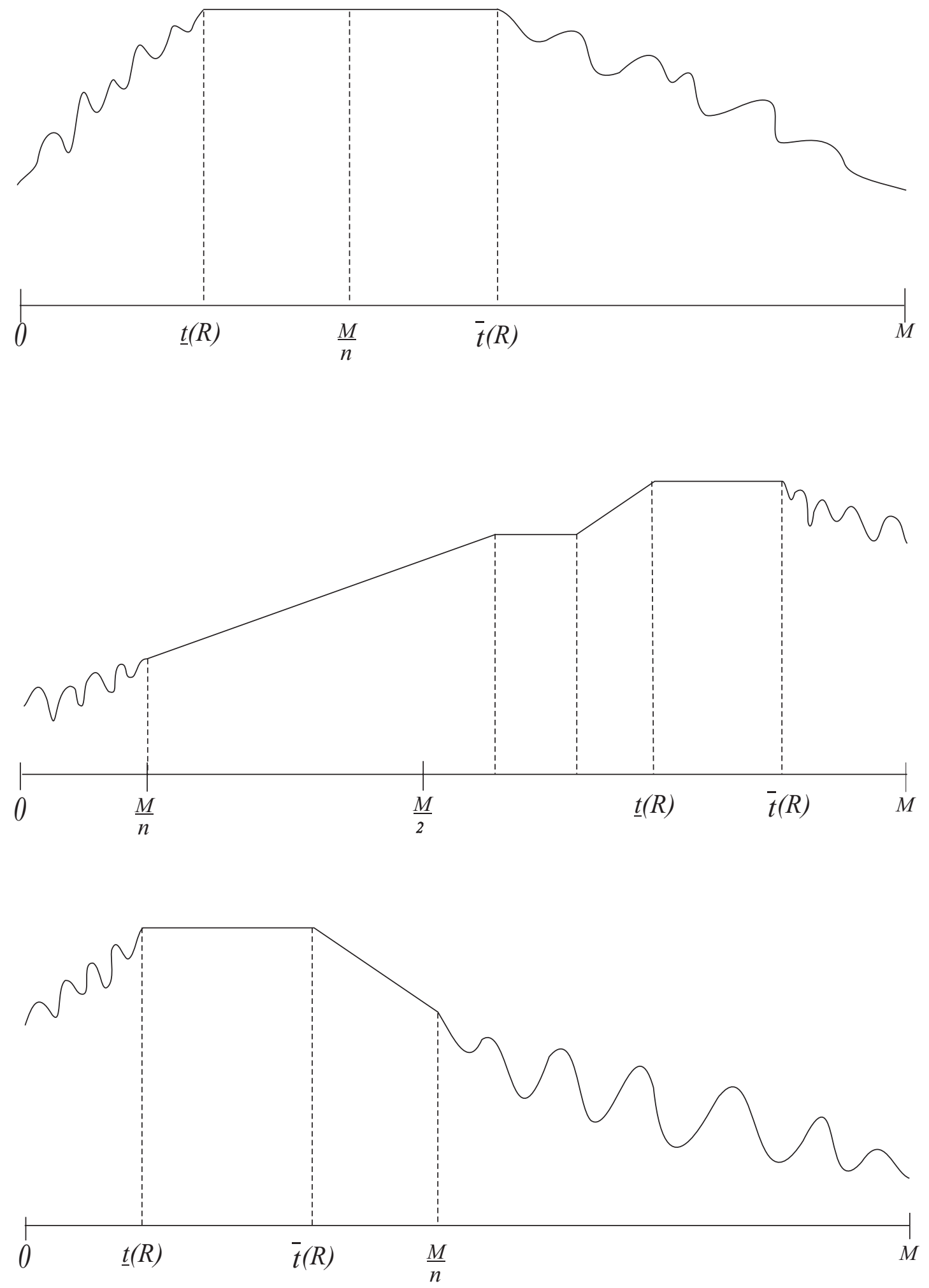

Figure 1 


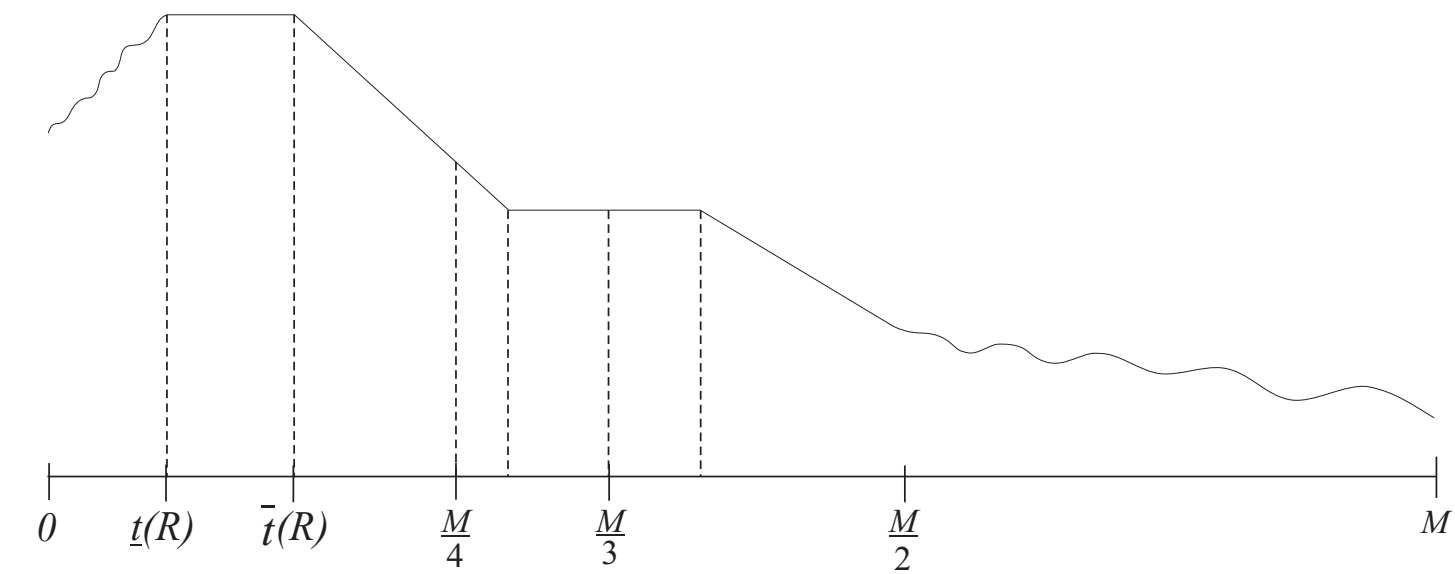

Figure 2 


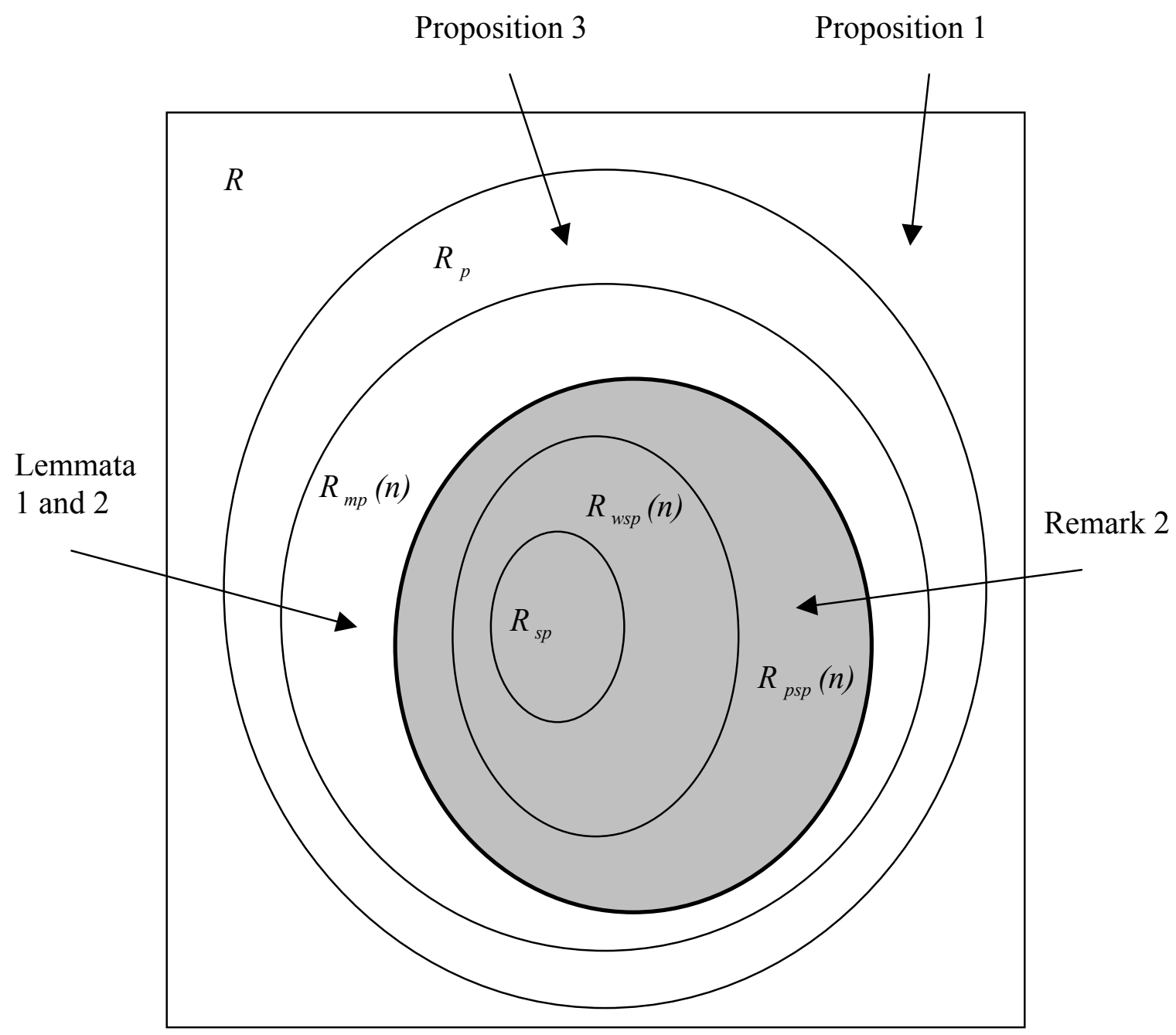

Figure 3 